\title{
Functional equivalence of the two sides of the human tongue
}

\author{
JAN H. A. KROEZE \\ Utrecht University, Utrecht 2501, The Netherlands
}

\begin{abstract}
Taste sensitivity to sucrose and $\mathrm{NaCl}$ of the left and right side of the human tongue was compared. The results did not show a significant difference when the data of the whole experimental group were taken together. Analysis of the individual results showed that there was one exception: A female subject showed a quality-specific difference between left and right. A speculative explanation is offered for this result.
\end{abstract}

In psychophysical or electrophysiological experiments where sapid stimuli are applied to one side of the tongue, the other side of the tongue may be used as a control. McBurney, Collings, \& Glanz (1973) did this when investigating the temperature dependence of the human behavioral taste response. This method is acceptable at least when the sides of the tongue are equal relative to the particular phenomenon under investigation. Although, to our knowledge, there is no direct experimental evidence available, it is generally assumed that the left and right sides' taste thresholds are the same.

Anatomical symmetry, as revealed by degeneration studies and electrophysiology (Ganchrow \& Erickson, 1972; Norgren, 1974; Norgren \& Leonard, 1973), does not imply functional symmetry, especially when taste functioning is not tested at a behavioral level. At a behavioral level, little is known about left-right relationships in taste. The only case where left-right differences were found was in a study of Kluyskens and Vandenhove (1969). In a clinical setting, they stimulated the chorda tympanic region of the human tongue electrically; they found that $35 \%$ of their subjects $(\mathrm{N}=117)$ showed a left-right sensitivity difference, the right side being more sensitive about twice as often as the left.

The experiment to be reported here was performed in order to compare the absolute sensitivity of the two sides of the tongue in human subjects when sapid stimuli are used.

\section{METHOD}

\section{Subjects}

Ten subjects (five male, five female) participated in the experiment. They were undergraduate, nonsmoking, right-handed psychology students. Their ages ranged between 20 and 24 years (mean: 20 years and 11 months). These 10 subjects were selected from 12 candidates on the basis of roughly equal thresholds.

We would like to thank Dr. D. H. McBurney for his advice on the construction of the tongue box.

\section{Stimuli}

For each subject, a perspex tongue box (Figure 1) that fitted each subject's tongue optimally was constructed. The box separated the left and right halves of the tongue. A small stream of distilled water continually flowed over the midregion of the tongue. Before use, the boxes were tested for leakage. In A and B (see Figure 1), the small low-pressure waterflow was introduced and one side of the tongue was stimulated with a red amarant solution. In this way, visual inspection of leakage was possible. The boxes turned out to be imperfect, and a tube had to be fitted through A and B with a small hole in it in front of the central gutter of the box. This modification meant that the waterstream could be led more directly to the midregion of the tongue. The stimuli were applied to the tongue by small polystyrene injectors which contained $1 \mathrm{ml}$ of stimulus liquid. The injectors were emptied at the location indicated by an asterix in Figure 1. The time between the onsets of two successive stimuli was fixed at $50 \mathrm{sec}$. Each stimulus was paired with a blank, which could follow or precede it. The time between stimulus and blank was as short as possible and never exceeded $1 / 2 \mathrm{sec}$. When subtracting stimulation duration from the total interval of $50 \mathrm{sec}$, an empty interval of about $45 \mathrm{sec}$ resulted, which is more than the time required for recovery of the sensitivity after adaptation (Hahn, 1934). Sodium chloride and sucrose of analytical grade purity diluted in distilled water were used as stimuli. The highest concentration of $\mathrm{NaCl}$ was $1.9 \times 10^{-1} \mathrm{~mol} / \mathrm{liter}$ and the highest sucrose solution was $3.8 \times 10^{-1} \mathrm{~mol} /$ liter. Starting with these solutions, stimulus ranges of 18 concentrations for each substance were obtained by diluting on a $2-\log$ base. All stimuli had a temperature of $22.5^{\circ} \mathrm{C}( \pm .4)$.

\section{Procedures}

Two different procedures were used. First, a crude whole-tongue threshold was determined by the up-down method described by

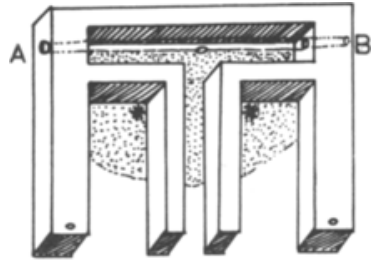

upper part

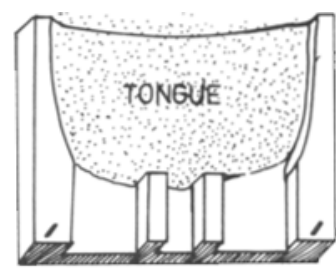

lower part
Figure 1. A schematic drawing of the tongue box used to separate left and right tongue halves. The dotted area shows the position of the tongue. 
Wetherill and Levitt (1965). All 18 concentrations were used in this test. On the basis of this crude threshold determination, a range of nine stimuli was selected for each subject in such a way that the preliminary threshold was in the middle. Ten out of the 12 subjects participating in this stage of the investigation turned out to be about equally sensitive, which meant that the threshold differences between them never exceeded two steps in the dilution range. The two remaining subjects, one male and one female, were so extremely insensitive that they had to be excluded for reasons of economy.

The lowest intensities selected for the next stage of threshold determination were $7.42 \times 10^{-4} \mathrm{~mol} /$ liter for $\mathrm{NaCl}$ as well as for sucrose. With the selected stimuli, thresholds were determined by the method of constant stimuli (D'Amato, 1970). This method was combined with a temporal two-alternative forced-choice procedure. Each trial consisted of the following series of events: (1) rinsing with distilled water (sipping method), (2) insertion of subject's tongue in stimulation box, (3) waiting until clock gives signal, (4) application of first stimulation (or blank), (5) application of second stimulation (or blank), (6) removal of tongue from stimulation box, and (7) registration of subject's response. The subjects indicated verbally which of the two stimuli presented immediately after each other was a blank (distilled water only). They were instructed to respond even if, in their opinion, the two numbers of a pair were equal. In such a case, they were told to make the best guess they could.

\section{Randomization}

Each series of nine stimuli was randomized 10 times. The reversed orders of these 10 series were also used. So a total of 20 replications of the stimuli was presented to each lateral side of the tongue. This was done for both substances in exactly the same way. All trials were randomized with respect to presentation order (blank or stimulus first).

Total participation for each subject was about $12 \mathrm{~h}$ divided into six 2-h sessions. Each session was subdivided into four 30 -min subsessions with a pause of 3 to $5 \mathrm{~min}$ in between. Stimulation locus was changed from one tongue half to the other after every 30-min subsession. Half of the subjects started with the left side of the tongue (two female and three male); the other half started with the right side (three female, two male). There was one 2-h sesson a day, and subjects came on alternating days. In the first three sessions, the detection threshold of $\mathrm{NaCl}$ was measured for half of the subjects, and in the last three sessions, the sucrose threshold was determined. For the other half of the subjects, this order was reversed. There were two identical ex-

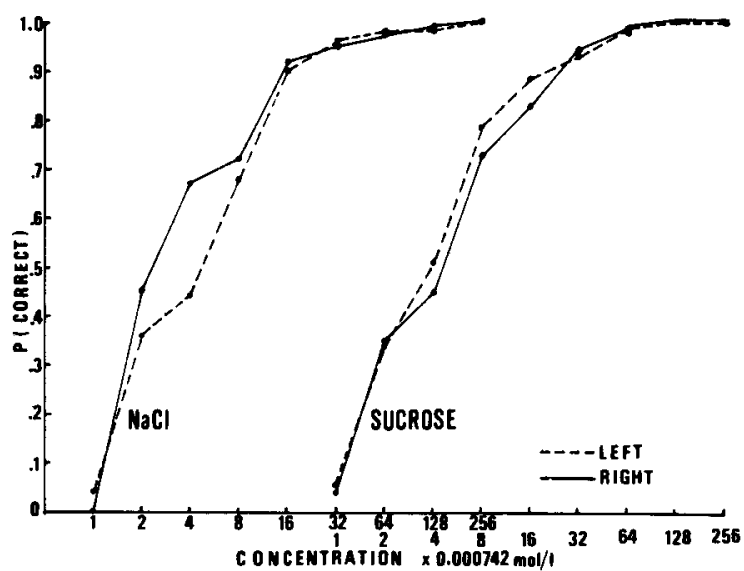

Figure 2. Threshold functions for $\mathrm{NaCl}$ and sucrose for different sides of the tongue. The unit on the abscissa is $\mathbf{. 0 0 0 7 4 2}$ $\mathrm{mol} / \mathrm{liter}$, which is the lowest concentration used (dotted lines, left; solid lines, right).
Table 1

Summary of Individual and Group Thresholds in mol/lifer

\begin{tabular}{cccccc}
\hline & \multicolumn{2}{c}{$\mathrm{NaCl}$} & \multicolumn{2}{c}{ Sucrose } \\
\cline { 2 - 3 } \cline { 5 - 6 } Sucrose & Left & Right & & Left & Right \\
\hline A & .00046 & .00121 & .00183 & .00141 \\
B & .00535 & $.01139^{*}$ & .00289 & .00277 \\
C & .00881 & .00496 & .00107 & $.01221 \dagger$ \\
D & .00303 & $.00120^{*}$ & .00420 & .00479 \\
E & .00636 & .00482 & .01272 & .00938 \\
F & .00112 & .00112 & .00184 & .00159 \\
G & .00650 & .00384 & .00208 & .00220 \\
H & .00176 & .00139 & .00281 & .00169 \\
I & .00078 & .00101 & .00132 & .00133 \\
J & .00403 & .00266 & .00619 & .00687 \\
Group & .00314 & .002266 & .00275 & .00314 \\
\hline
\end{tabular}

Note-These thresholds were determined by fitting sigmoid curves to the chance-corrected threshold data and then determining the stimulus concentration at the $p=.50$ point. According to a binomial criterion, there are only three significant differences. ${ }^{*} p<.05 . \quad t p<.0001$

perimental setups, so two subjects could be run simultaneously. For each setup, there were two experimenters. One prepared the stimuli, while the other presented them to the subject.

\section{RESULTS}

For each subject, the cumulative frequency distribution was calculated after correcting the data for chance. The group results can be seen in Figure 2 . The individual results are summarized in Figure 3. Table 1 gives a summary of all thresholds. In order to obtain the values in this table, sigmoid curves were fitted to the chance-corrected threshold data. This was done by a FORTRAN program called SIGMPLOT (Drake, 1975). From these sigmoid functions, the $p=.50$ points are listed in the table.

For each subject, an individual score for both sides of the tongue was calculated. This score was defined as the number of correct detections after corrections for chance. Testing the differences between left and right for the whole group with a $t$ test for correlated samples revealed no differences $(\mathrm{NaCl}: \mathrm{t}=.42, \mathrm{df}=9, \mathrm{p}>.20)$.

On the basis of the group results, a left-right sensitivity difference is not likely. Inspection by eye reveals that in the case of $\mathrm{NaCl}, 4$ out of 10 subjects do not show identical threshold functions for left and right (Figure 3: Subjects B, C, D, and G). For sucrose, this is 3 out of 10 (subjects $C, E$, and $\mathrm{H})$. For these pairs of threshold functions, the difference between right and left is statistically tested in the following way. The frequencies of the various intensity levels were totaled for each side of the tongue. The $100 \%$ and $0 \%$ levels were only once included in this count, because these levels do not contain threshold information. If left and right are equal, then half of the total sum should be expected 

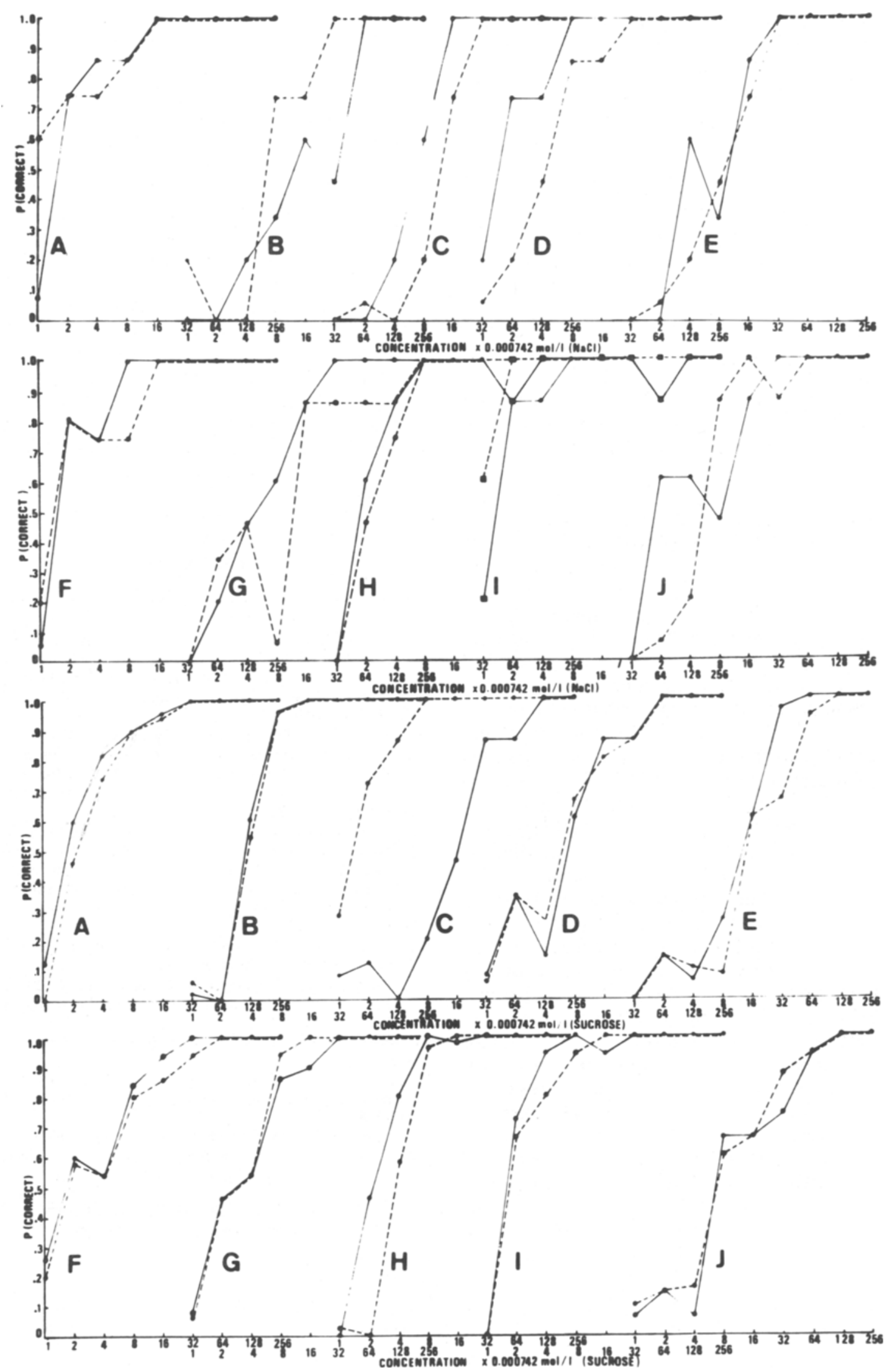

Figure 3. Individual threshold functions of the left and right tongue halves (dotted lines, left; solid lines, right). 
to fall on one tongue side and the other half of the scores on the contralateral side. This was tested by applying a binomial criterion. Only two subjects showed a difference that was significant at $5 \%$ or lower. One male (Subject D) showed a difference that was significant at the $5 \%$ level: He was more sensitive to $\mathrm{NaCl}$ when stimulated on the right side of the tongue. The other subject (C), a female, showed a strong lateral dominance of the left tongue half when stimulated with sucrose $(p<.0001)$.

\section{DISCUSSION}

When searching for differences in 20 samples, one should not be surprised to find one or two results being significant at the $5 \%$ level. But the extreme $p$ value found in Subject $C$ leads to the conclusion that lateral dominance, at least when tasting sucrose, might exist in certain subjects. This exception is puzzling. Repeated sensitivity measurements constantly gave the same results. Thus, there is no use in explaining it as an experimental artifact. Neither is it likely that it can be contributed to chorda tympani damage as a result of middle ear traumata, as there were no such facts in the subject's history. Even if there had been chorda tympani damage, it would not be understandable how this could have selectively diminished sucrose sensitivity without affecting $\mathrm{NaCl}$ sensitivity at the same time.

A speculative suggestion may be that although the input from both sides into the thalamic area and from there up into the cortical projection zones may be equal for certain substances, it may not be equal for other substances. Degeneration studies cannot give the answer here, because experimental lesions of complete fiber arrays may also destroy the selective mechanism involved. Electrophysiological studies could perhaps provide some information. In those pairs in which the taste is presented first, the following blank may take on a taste (Bartoshuk, 1968, 1974). Although the stimulation times were probably too short to induce this so-called water taste, it was interesting to examine it by comparing the number of incorrect responses of both orderings of stimulus and blank. These were essentially the same for supra- and for subthreshold $\mathrm{NaCl}$ and sucrose concentrations. Thus, the thresholds probably are not water thresholds.

As in olfaction (Koelega, 1979), the results of this experiment do not permit the conclusion that, in taste, lateral dominance is a generally occurring phenomenon. In group results, the probability that lateral dominance seriously affects the data when one side of the tongue is used as a control while the other side is stimulated, is not high. However, it is advisable to check it in some way if only a few subjects are used; exceptions to the rule may occur. Where lateral dominance occurs on the left side as well as on the right side of a perceptual system, a group approach may have the disadvantage of obscuring the phenomenon. Therefore, an additional analysis of individual results may be recommended. In taste, the left tongue side of one individual may be the other's right side.

The picture is complicated by the possibility that lateral differences may be located at different levels in the taste system. Although animal research may give important information relative to the anatomical and physiological comparison of left and right, human research may remain of special importance, because lateral specialization is far more striking in humans than in other mammals.

\section{REFERENCES}

D'Amaтo, M. R. Experimental psychology: Methodology, psychophysics and leaming. New York: McGraw-Hill, 1970.

Bartoshuk, l. M. Water taste in man. Perception \& Psychophysics, 1968, 3, 69-71.

BartoshuK, L. M. NaCl thresholds in man: Thresholds for water taste or $\mathrm{NaCl}$ taste? Joumal of Comparative and Physiological Psychology, 1974, 87, 310-325.

DRAKE, B. A FORTRAN program, "SIGMPLOT," for fitting sigmoid curves to threshold data and for plotting the result. Chemical Senses and Flavor, 1975, 1, 519-531.

GANChRow, D., \& ERICkson, R. P. Thalamocortical relations in gustation. Brain Research, 1972, 36, 289-305.

Hahs, H. Über die Adaptation des Geschmackssinnes, Zeitschrift für Sinnesphysiologie, 1934, 65, 105-145.

KuUYskens, P., \& Vandenhove, P. L'électrogustométrie, test valable dans l'appréciation de la fonction de la corde du tympan et de sa lésion postopératoire. Acta Oto-Rhino-Laryngologica Belgica, 1969, 23, 23-32.

KoeleGA, H. S. Olfaction and sensory asymmetry. Chemical Senses \& Flavour, 1979, 4, in press.

McBurkey, D. H., Collings, V. B., \& Glanz, L. M. Temperature dependence of human taste responses. Physiology \& Behavior, 1972, 11, 89-94.

NoRgren, R. Gustatory afferents to ventral forebrain. Brain Research, 1974, 81, 285-295.

Norgren, R., \& Leonard, C. M. Ascending central gustatory pathways. Journal of Comparative Neurology, 1973, 150, 217-238.

Wetherill, G. B., \& LevitT, H. Sequential estimation of points on a psychometric function. British Joumal of Mathematical and Statistical Psychology, 1965, 18, 1-10.

(Received for publication January 5, 1978; revision accepted November 20,1978 .) 